\title{
FROM THE TIP OF THE AGB TOWARDS A PLANETARY: A HYDRODYNAMICAL SIMULATION
}

\author{
D. SCHÖNBERNER, M. STEFFEN, J. STAHLBERG, \\ K. KIFONIDIS AND T. BLÖCKER \\ Astrophysikalisches Institut Potsdam \\ D-14482 Potsdam, Germany
}

\begin{abstract}
We present a first exploratory investigation of the dynamical evolution of a dusty stellar wind envelope along the upper AGB and its transformation into a planetary nebula. We find the existence of AGB stars with detached shells to be a natural consequence of the mass-loss variations during a thermal pulse. It is also demonstrated that due to the large dynamical effects caused by the ionizing radiation field and the fast wind of the central star, it is impossible to deduce the AGB mass loss history from the planetary's density and velocity distribution. The structure of the halo, however, is still determined by the AGB mass loss history. The rapid decline of mass loss expected in the aftermath of thermal pulses leads to extended shells of low densities and explains halos with sharp boundaries.
\end{abstract}

\section{Introduction}

Recent attempts to model the development of planetary nebulae with evolving central stars by solving the coupled equations of radiation hydrodynamics have been remarkably successful, but so far all of these studies have started with initial structures which are exclusively based on guesses. This fact is, of course, just a reflection of our ignorance of the dynamical behavior of the dusty wind envelopes of AGB stars. Knowledge of the density, velocity, chemical, and ionization structures of stellar wind envelopes when the star leaves the AGB appears to us of paramount importance for understanding the evolution of proto- and well-developed planetaries as well as for the interpretation of observations. Important questions that have to be investigated are:

1. the short and long term behavior of AGB envelopes subject to stellar mass-loss variations on time scales smaller than the flight time of a gas parcel; 
2. the final structures after the end of the AGB evolution when heavy mass loss ceases and the remnant starts to increase in $T_{\text {eff }}$;

3. whether a well-developed planetary-nebula shell still contains information about the mass loss history during the AGB evolution.

It is the purpose of this contribution to address these questions and to give first answers. We present here the very first attempt to compute the dynamical response of a stellar wind envelope for the last several hundred thousand years of AGB evolution and the following 5000 years of postAGB evolution. Based on self-consistent stellar evolution calculations, the adopted mass loss rate varies as a function of the stellar parameters. It should be emphasized that although the mass-loss law has not been derived from first principles (for details see Blöcker 1995), the stellar evolution calculations and the computations of the response of the envelope presented here are internally consistent once the physical details of the mass loss have been specified (see below).

\section{Evolution towards the Tip of the AGB}

Figure 1 illustrates the temporal evolution of luminosity and mass-loss rate for the last $\sim 350000$ years on the AGB and the following few thousand years of the post-AGB evolution of a solar composition stellar model computed by Blöcker (1995). Mass loss reduces the initial mass of $3 M_{\odot}$ to a final mass of $0.605 M_{\odot}$. The stellar outflow is assumed to be spherically symmetric, and the equations of hydrodynamics are solved for the gas and dust components, coupled by momentum exchange due to dust-gas collisions. The code is a modification of the one developed by Yorke \& Krügel (1977) and makes use of the following simplifications:

1. Radiation transfer is considered only for the dust component, i.e. exchange of photons between dust grains and the gas is neglected.

2. The dust temperature is computed from radiative equilibrium, and the gas component (neutral hydrogen) is assumed to have the same (local) temperature. This is justified since we are not interested in computing line emissions, and since the influence of the gas-pressure gradient on the outflow velocity turns out to be quite small.

3. The dust component consists of single-sized grains, based on either oxygen or carbon chemistry, and a fixed dust-to-gas ratio is assumed at the condensation point.

A more detailed description of this fully implicit radiation hydrodynamics code, including comparisons with solutions for stationary outflows given in the literature, will be published in the near future.

The new approach applied here is to use time-dependent values of stellar mass, luminosity, effective temperature and the resulting variable mass loss 

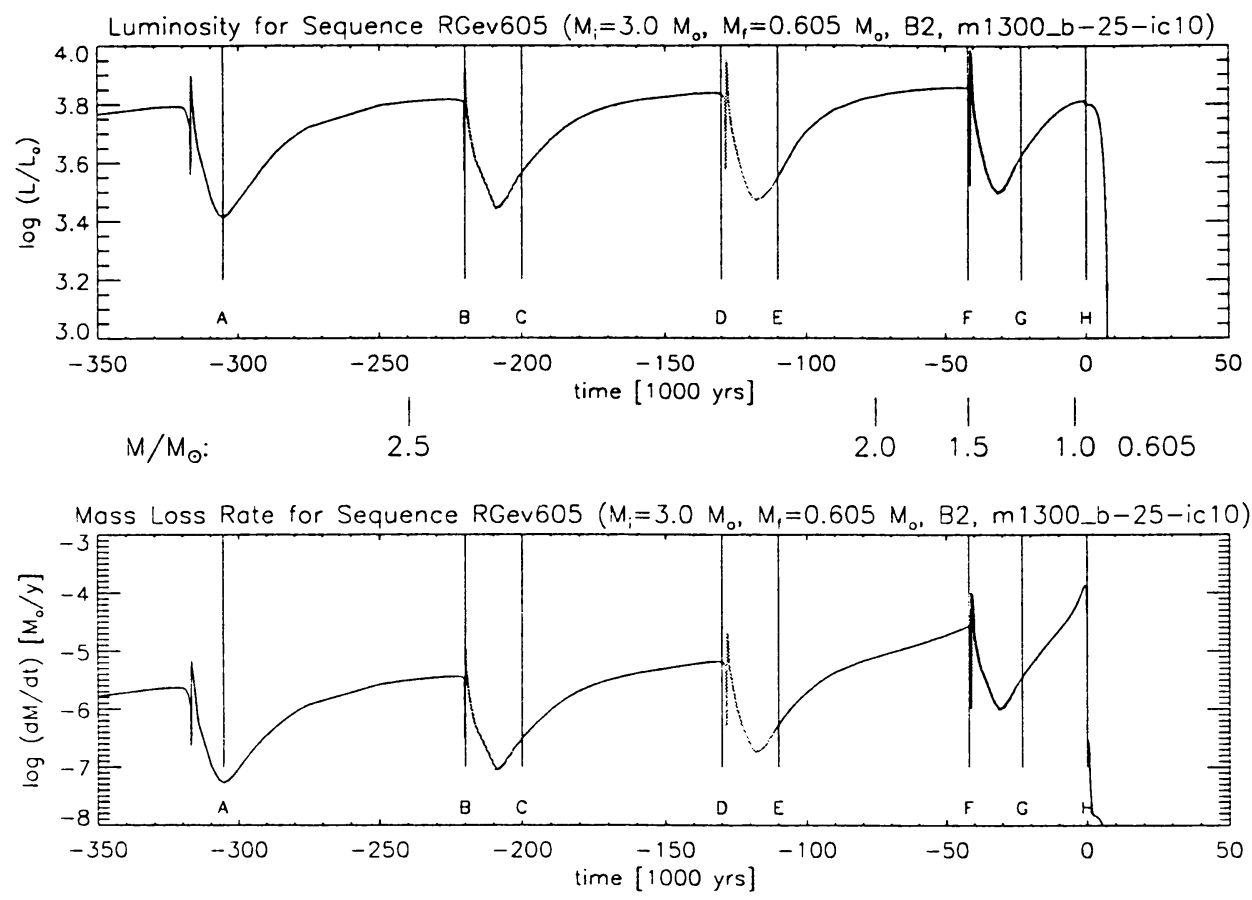

Figure 1. Luminosity vs. time (top) and mass loss rate vs. time (bottom) for the last four thermal-pulse cycles of a $3 M_{\odot}$ model sequence, after Blöcker (1995). Time zero marks the beginning of the model's post-AGB evolution. The current stellar mass is indicated between the two panels. Labels $\mathrm{A}$ to $\mathrm{H}$ are referred to in the text.

(as shown in Fig. 1) at the inner boundary of our numerical grid, with a constant flow velocity equaling the local sound velocity $\left(\sim 3 \mathrm{~km} \mathrm{~s}^{-1}\right)$. The radiation pressure on the grains and the momentum exchange with the gas leads to an acceleration of the material with typical final outflow velocities around 10 to $15 \mathrm{~km} \mathrm{~s}^{-1}$, as are also observed. We started the computations at point A in Fig. 1 and continued through three consecutive thermal pulses until the end of the AGB phase is reached $(\mathrm{H})$.

The pronounced variations of the mass loss rates during the thermal pulses (sections B-C, D-E and F-G in Fig. 1) lead to large deviations of density and velocity from the cases of stationary outflows. Correspondingly, the emerging spectral energy distributions (SEDs) change with time as demonstrated in Figure 2 for the first computed pulse (B-C). Dust grains with a radius of $0.05 \mu \mathrm{m}$ are made of amorphous carbon and the dust-to-gas mass-density ratio is assumed to be $1.5 \times 10^{-3}$. The time-dependent models produce drastic changes of the spectral energy distributions on even rather short time scales (e.g. $\sim 300$ years between $t 2$ and $t 3$ ) which are solely due to the large variations of the mass-loss rate and the corresponding 

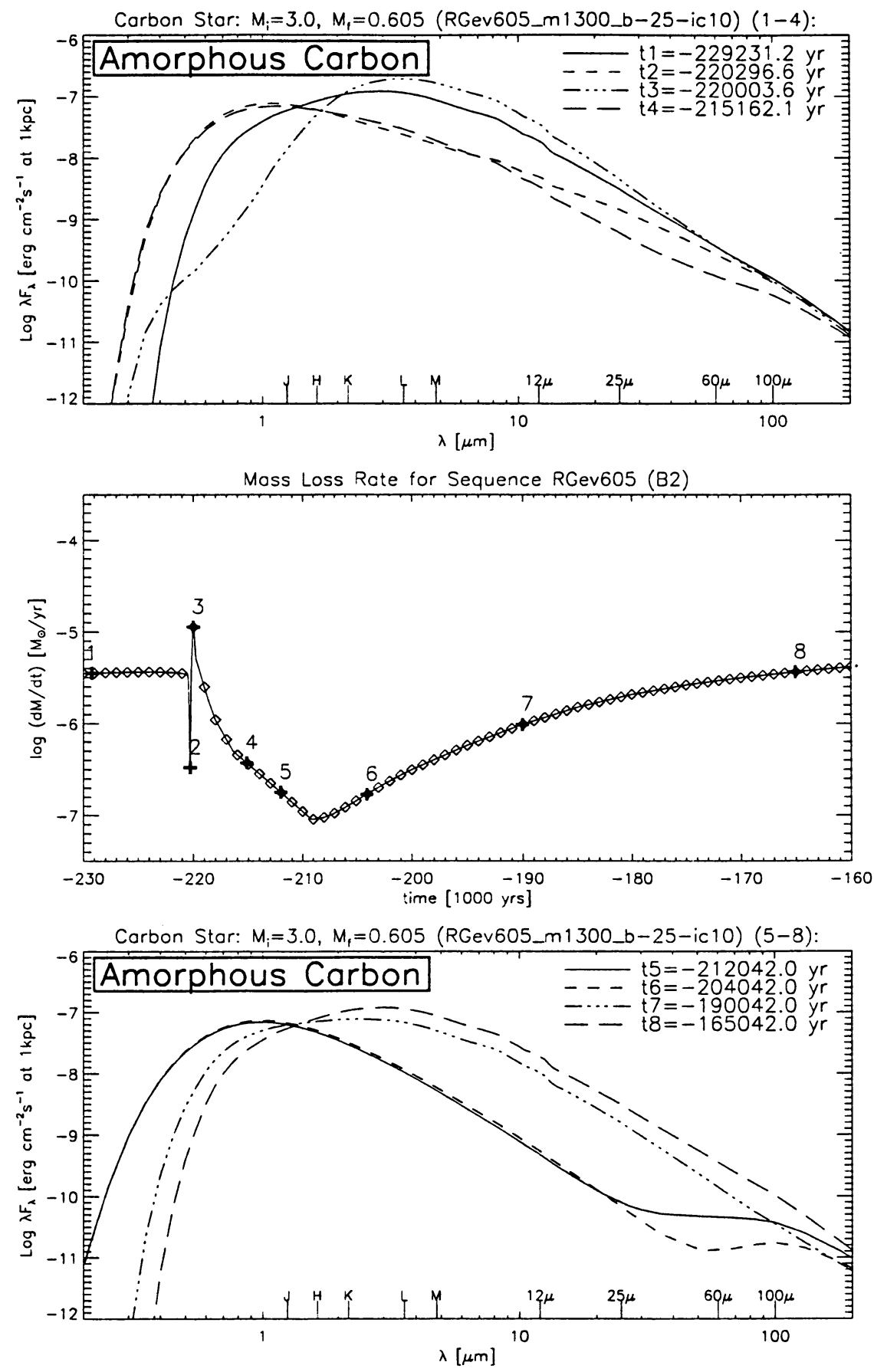

Figure 2. Spectral energy distributions (upper and lower panels) at selected times for a carbon star that experiences a thermal pulse (middle panel). The infrared and IRAS pass bands are indicated. For further details see text. 


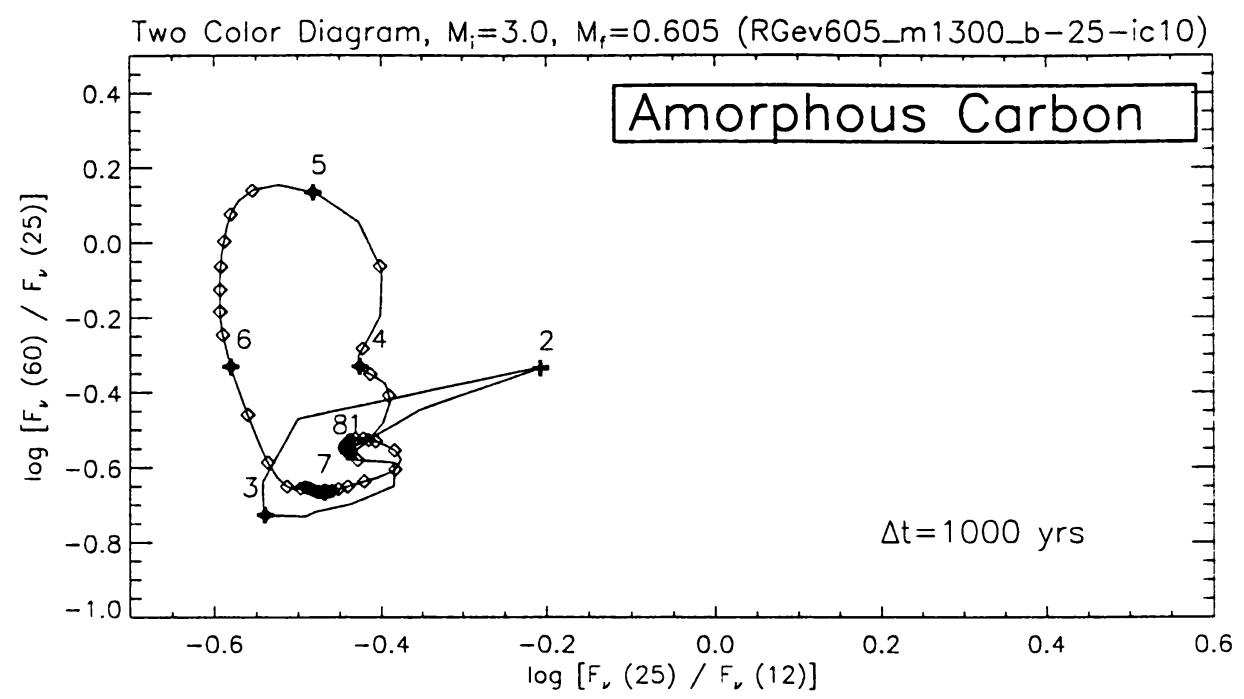

Figure 3. IRAS two-color diagram of the model sequence shown in Fig. 2, with numbered + signs indicating the same times as before. Diamonds outline the time evolution in steps of $\Delta t=1000$ years. Colors at times 1 and 8 are identical.

deviations from stationarity. Although the mass-loss rates are practically equal at times $t 2$ and $t 4$, there are considerable differences in the resulting far-IR emission. The same holds for times $t 5$ and $t 6$.

During the whole mass loss minimum the dust shell is optically thin and the star is completely visible $(t 2, t 4, t 5, t 6)$. The large far-IR excess is due to a cool detached dust shell containing matter which has been expelled well before the onset of the thermal pulse. Objects with far-IR excesses are known (e.g. Chan \& Kwok 1988; Zijlstra et al. 1992; Kerschbaum \& Hron 1996; Olofsson et al. 1996), and our computations give a quite natural explanation in terms of thermally pulsing AGB stars. Later the envelope becomes optically thick again $(t 7, t 8)$ and can be approximated by stationary flow solutions.

The changes in spectral appearance shown in Fig. 2 are responsible for the object's movement in the two-color diagram as shown in Figure 3. Similar loops can be found by coupling envelopes with silicate grains to the stellar parameters.

Several remarks are in order. ( $i$ ) In addition to the loops, our dynamical envelopes produce some intrinsic scatter in the two-color diagram, solely due to the deviations from stationary conditions. (ii) As the consequence of a thermal pulse, objects on the upper AGB can develop detached dust shells and spend about $10 \%$ of their time away from the main two-color relation of dusty AGB stars. Our computed SEDs are in good agreement with 
corresponding observations, which may, however, be disturbed by cirrus contamination in some cases. In contrast to Ivezić \& Elitzur (1995), we believe that the modeling of dusty envelopes around AGB stars as stationary outflows is not justified a priori and may lead to erroneous interpretations!

\section{Evolution towards a Planetary Nebula}

In the mass-loss modeling by Blöcker (1995) it has been assumed that the mass loss rapidly decreases with the radial fundamental pulsational period of the remnant until the Reimers (1977) rate is reached at $P=50$ days. This rate is then kept until the remnant becomes hot enough for the application of the theory of radiation-driven winds (Pauldrach et al. 1988) which provides mass-loss rate and wind speed. A more detailed description of the physical model used for the post-AGB evolution and how mass loss rate and wind speed vary in the course of the evolution can be found in Marten \& Schönberner (1991).

The kinematic structure of the last model of the AGB evolution, computed at $t=0$, is shown in the upper panel of Figure 4. The density structure is clearly different from the usual assumption of an $r^{-2}$ law: This model reflects the last 100000 years of AGB evolution, showing a large density dip caused by the last thermal pulse about 30000 years ago (cf. Fig. 1), but a rapid density increase inwards $\left(\propto r^{-3}\right)$ due to the increasing mass-loss rate. Further inwards the density increase flattens somewhat $\left(\propto r^{-1}\right)$. The outflow velocity is rather constant except for a slight decrease caused by the luminosity dip of the last pulse.

This model is then used as input for a newly developed, explicit radiation hydrodynamics code based on a second-order Godunov-type advection scheme (Walder, private communication) in order to follow the evolution into the domain of planetaries. The radiation part of this code considers time-dependent ionization, recombination, heating and cooling for up to nine elements (Marten \& Szczerba 1997). Here we have used the elements $\mathrm{H}, \mathrm{He}, \mathrm{C}, \mathrm{N}, \mathrm{O}$ and $\mathrm{Ne}$ with all of their ionization stages. More details of our dynamical modeling of planetaries will be published in the near future.

The lower panel of Fig. 4 gives an example of a well-developed nebular structure of intermediate size. The fast but tenuous stellar wind terminates through a strong inner shock (near $r=3 \times 10^{16} \mathrm{~cm}$ ), and the high pressure of this shocked wind material compresses the inner parts of the planetary to a high-density, low-velocity shell (just beyond C in Fig. 4). The outer shell (just inside B) has much lower density, contains matter with larger velocities (up to $30 \mathrm{~km} \mathrm{~s}^{-1}$ ) and snowplows through the halo which is still the original but ionized AGB wind. Please note that the outer shock front (B), which defines essentially the observed outer rim, expands with an even higher 

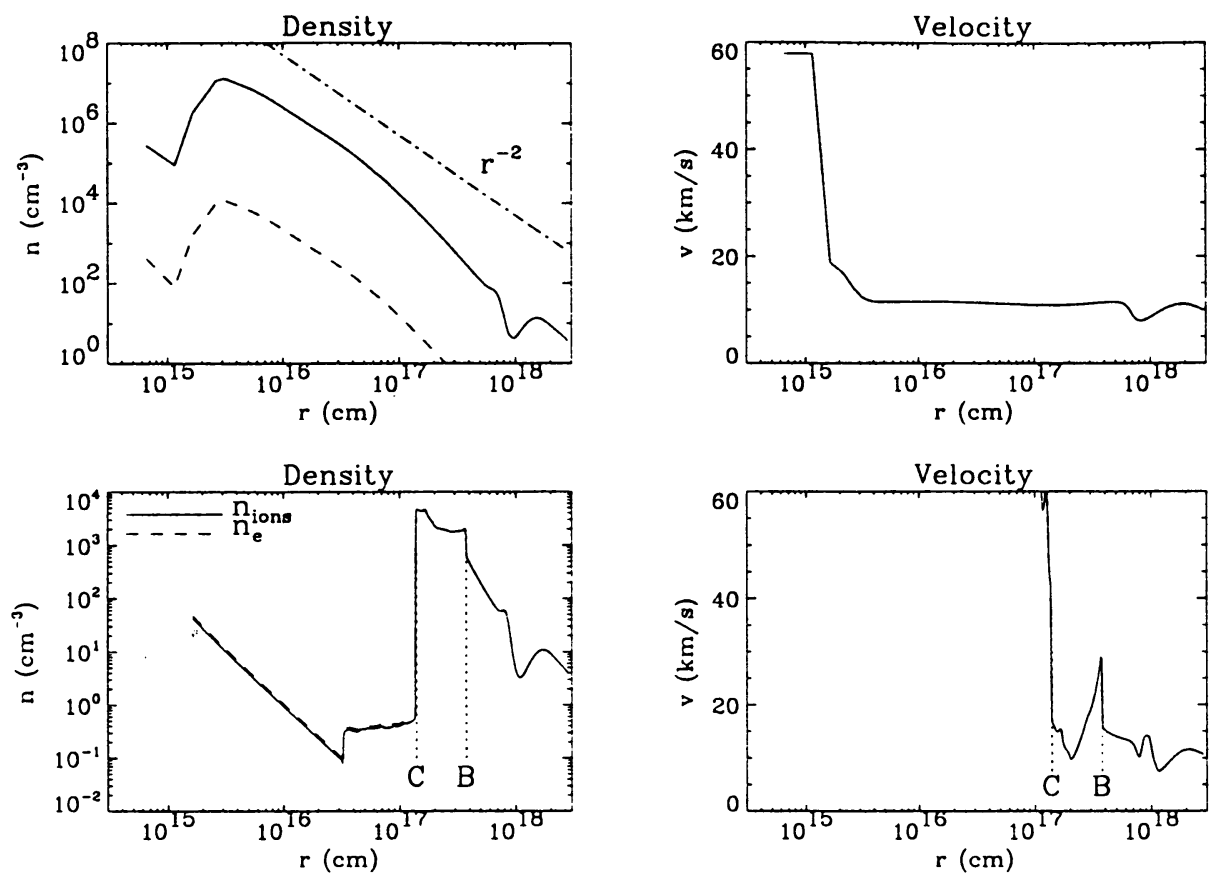

Figure 4. Upper panel: Kinematic structures of the initial model $(t=0)$ for post-AGB evolution, with central star parameters $L=6310 L_{\odot}$ and $T_{\text {eff }}=6053 \mathrm{~K}$. Lower panel: 4603 years later: now $L=5602 L_{\odot}$ and $T_{\text {eff }}=89851 \mathrm{~K}$. The planetary proper is the region between the contact discontinuity $(\mathrm{C})$ and the outer shock $(\mathrm{B})$.

velocity of about $40 \mathrm{~km} \mathrm{~s}^{-1}$. Since the whole grid is completely ionized, such a planetary would be called "density bounded." Morphologically one would speak of a well-developed double-shell structure (Chu et al. 1987).

It is clear from Fig. 4 that the kinematic structure of the planetary proper (between $\mathrm{C}$ and $\mathrm{B}$ ) has no resemblance whatsoever to the mass loss history that originally led to its existence. In particular, the double-shell structure has absolutely nothing to do with recurrent helium shell flashes, as has been proposed by Tuchman \& Barkat (1980). Rather, this structure is a consequence of photoionization and wind interaction and appears also for simpler initial models, for instance for an $r^{-2}$ density law and also for the "superwind" model of Marten \& Schönberner (1991).

The situation is different for the halo: The density dip (near $r=10^{18} \mathrm{~cm}$ ) caused by the last thermal pulse is still existent, and also the velocity field has not changed much. This low-density region has observational consequences for the surface-brightness distribution of, for instance, $\mathrm{H} \beta$ (Figure 5). The halo appears to have a rather sharp boundary, and one may speak, in analogy, of a "density bounded" halo, which is typical for the few 


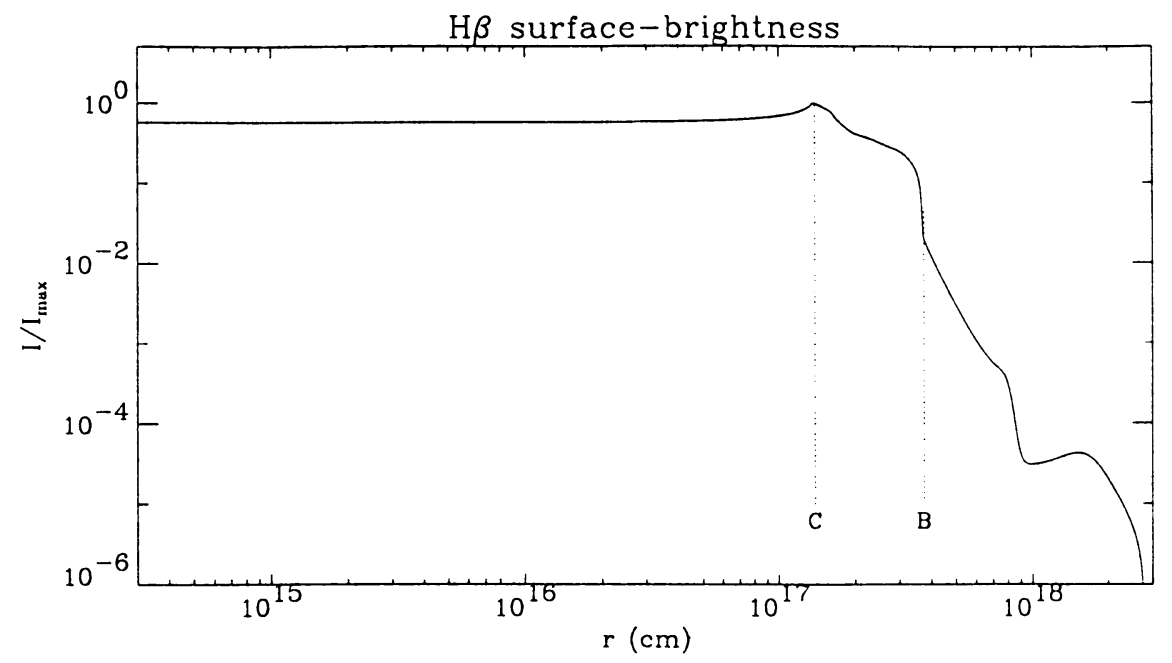

Figure 5. Surface-brightness distribution in $\mathrm{H} \beta$ of the nebular model shown in the lower panel of Fig. 4. Again, the positions of the outer shock (B) and the contact discontinuity (C) are indicated. Note the sharp outer rim of the halo near $r=10^{18} \mathrm{~cm}$.

known cases (Chu 1989). Our model shown in Fig. 5 resembles remarkably well the $\mathrm{H} \alpha$ image of NGC 6826 (cf. Plait \& Soker 1990). Actually, NGC 6826 is classified as a triple-shell planetary (Chu et al. 1987), and our interpretation would be that these relatively rare objects are typical double-shell objects where the halo is visible as a third shell.

Part of this work was supported by the German Space Agency, DARA, under grant WE2 50 OR 9411.

\section{References}

Blöcker, T. 1995, $A \& A, 297,727$

Chan, S. J. \& Kwok, S. 1988, ApJ, 334, 362

Chu, Y.-H. 1989, in IAU Symp. 131: Planetary Nebulae, ed. S. Torres-Peimbert (Kluwer), p. 105

Chu, Y.-H., Jacoby, G.H. \& Arendt, R. 1987, ApJ Supp., 64, 529

Ivezić, Ž. \& Elitzur, M. 1995, $A p J, 445,415$

Kerschbaum, F. \& Hron, J. 1996, $A \& A, 308,489$

Marten, H. \& Schönberner, D. 1991, $A \& A, 248,590$

Marten, H. \& Szczerba, R. 1997, $A \& A$, 325, 1132

Olofsson, H., Bergman, P., Eriksson, K. \& Gustafsson, B. 1996, A\&A, 311, 587

Pauldrach, A., Puls, J., Kudritzki, R.P., Méndez, R. H. \& Heap, S. R. 1988, $A \& A, 207$, 123

Plait, P. \& Soker, N. 1990, AJ, 99, 1883

Reimers, D. 1977, in Problems in Stellar Atmospheres and Envelopes, ed. B. Baschek, W. H. Kegel and G. Traving (Springer), p. 229

Tuchman, Y. \& Barkat, Z. 1980, ApJ, 242, 199

Yorke, H. W. \& Krügel, E. 1977, $A \& A, 54,183$

Zijlstra, A., Loup, C., Waters, L.B.F.M. \& de Jong, T. 1992, $A \& A$, 265, L5 


\section{Discussion}

Dorfi: What is your argument for using the mass-loss rate of Blöcker also during the thermal pulse phases?

Schönberner: Blöcker developed his mass-loss formula because, at that time, Bowen's calculations were the only ones available that considered radial pulsations together with radiation pressure on grains. We used Blöcker's prescription in the dynamical calculations presented here in order to keep the internal consistency between mass loss and stellar evolution. A detailed treatment of dust formation together with a self-consistent determination of mass-loss rates along the AGB is a tremendous amount of work which, however, certainly has to be done.

Wagenhuber: Depending on the mass-loss rate dependence on the effective temperature, the time scales for the transition from the AGB to $\sim 10^{4} \mathrm{~K}$ can be very different, so that cores of higher mass may mimic the evolutionary timescales of lower-mass cores.

Schönberner: In the model of Blöcker, it is assumed that the high AGB mass-loss rate declines when the stellar pulsational period decreases from 100 to 50 days. This always ensures that the transition towards the PN region is rather fast. This treatment seems to be in rough agreement with the observations since none of the observed post-AGB stars appears to be cooler than $5000 \mathrm{~K}$, as is also predicted by Blöcker's models. 